\title{
The concentration of calcium ions and of free electrons in a meteor coma ${ }^{\star}$
}

\author{
P. B. Babadzhanov and G. I. Kokhirova
}

\author{
Institute of Astrophysics, Tajik Academy of Sciences, and Isaac Newton Institute of Chile, Tajikistan Branch, \\ Bukhoro Str. 22, Dushanbe 734042, Tajikistan \\ e-mail: [pbb;gulya]@ac.tajik.net
}

Received 4 November 2003 / Accepted 23 April 2004

\begin{abstract}
Prism spectra are studied of eight bright meteors, belonging to different showers and photographed by small cameras in the Gissar observatory of the Institute of Astrophysics, Tajik Academy of Sciences. Monochromatic light curves for the $\mathrm{H}$ and $\mathrm{K}$ spectral lines of the ionized calcium doublet are constructed and from the ratio of their intensities at selected points along the meteor trajectories the column $N_{\text {CaII }}$ and the volume $n_{\text {CaII }}$ concentrations of calcium ions are determined. The obtained values $N_{\text {CaII }}=10^{16}-10^{18} \mathrm{~cm}^{-2}$ and $n_{\text {CaII }}=10^{13}-10^{15} \mathrm{~cm}^{-3}$ are in good agreement with the estimates of calcium abundance obtained by other methods and confirm that there is almost full ionization of calcium atoms in meteor comae. The volume concentration of free electrons $n_{\mathrm{e}}=4 \times 10^{13} \mathrm{~cm}^{-3}$ is defined, and the excitation temperature is calculated along the meteor paths.
\end{abstract}

Key words. meteors, meteoroids

\section{Introduction}

The determination of the concentration of atoms and ions of chemical elements is the main problem of the quantitative analysis of meteor spectra, since makes it possible to determine the chemical composition and type of the material of the meteoroids, namely, the quantitative abundances of the constituent elements and so to establish to what degree the minor bodies of the Solar system are similar, because it is now widely accepted that between asteroids, comets and meteoroids there exists a genetic interrelation.

Different methods of quantitative analysis of meteor spectra have been developed and used by researchers. The main difficulties of this analysis are that the radiation of a meteor coma is not in the thermal equilibrium state, and the radiating volume is not optically thin. Therefore, it is necessary to take into account the self-absorption in the spectral lines, because neglecting the self-absorption leads to underestimation of the abundances of the pertinent elements.

The method of the emission curve of growth (ECG), which is based on the assumption of local thermal equilibrium in the radiating volume, and takes into account the self-absorption in spectral lines, is well known in meteor spectroscopy. But for using it to construct the full emission curve of growth we need the presence of a sufficient number of multiplets for each element in the meteor spectrum. This condition is realized extremely

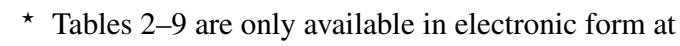
http://www.edpsciences.org rarely. Even for the grating spectrum of the -11 absolute magnitude fireball Ceplecha (1964) determined the absolute abundances of elements FeI, CaI, MgI, MnI, CrI, NiI, CoI, NaI, AlI, SiI and of CaII ions, but for the construction of an ECG only 70 iron lines were available. The ECG method cannot be used for investigating prism spectra of fainter meteors, obtained with lower dispersion.

Millman (1933, 1935, 1972) made a valuable contribution to the determination of the content of chemical elements in meteor spectra. For this purpose he used both grating and prism spectra, but he confined himself to qualitative analysis only, i.e. the identification of lines, and the description and classification of meteor spectra. The investigations of meteor spectra by Blajko (1932), Cook et al. (1971), Ceplecha (1965, 1967, 1971), Ceplecha \& Rajchl (1963), Rajchl (1961, 1974), Halliday (1961, 1987), Harvey (1971, 1973a, 1973b), Millman et al. $(1961,1971)$ were also concerned with qualitative analysis and some aspects of quantitative analysis.

To estimate the concentration of atoms in a meteor coma, Nagasawa (1978) used the method of Harvey (1973b), which is based on the assumption of thermal equilibrium in the meteor coma, and estimated the relative numbers of atoms FeI, NaI, MgI, SiI, MnI, CoI and of ions CaII and SiII for two Leonids, but for all this he also used grating spectra.

Investigating the grating spectra of bright meteors and fireballs Borovička (1993), and Borovička \& Betlem (1997) identified the lines in these spectra, and by means of various methods 
determined a number of the physical characteristics of meteors followed by physical interpretation of the obtained data.

It should be noted once more that for qualitative and quantitative analyses of meteors, different authors used grating spectra obtained with high resolution and dispersion from 11 to $50 \AA$ per mm, which typically show a large number of clearcut emission lines of both neutral and ionized elements.

It may be concluded that for the determination of the quantitative abundances of one or the various elements, researchers of meteor spectra used different methods, but these methods are almost never applicable to prism spectra because of these have a lower dispersion and, as a consequence, they only show a small number of lines available for measurements.

However, there is a possibility to use the rich observational material of prism spectra for the development of quantitative meteor spectroscopy, applying the methods applicable in stellar spectroscopy in the analysis of meteor spectra.

\section{Determination of the calcium ion concentration and the excitation temperature in a meteor coma}

In the present work on the determination of the calcium ion column concentration $N_{\text {CaII }}$ we have used the method, presented by Viazanitsin (1947) for spectrophotometric study of the solar prominences. This method is based on the assumption of local thermodynamic equilibrium in the radiating volume. We cannot take the influence of non-equilibrium radiation into account because of the absence of a complete theory. So, in the first approximation, all investigations are based on the assumption of local thermodynamic equilibrium (e.g. Millman 1935; Ceplecha 1964, 1965; Nagasawa 1978; Borovička 1993; Borovička \& Betlem 1997).

As was shown by Viazanitsin (1947), the ratio of the intensities of the $\mathrm{H}$ and $\mathrm{K}$ emission lines of ionized calcium (CaII) depends on the number of calcium ions $N_{\text {CaII }}$ in the column of surface area $1 \mathrm{~cm}^{2}$ along the line of sight (i.e. the column density) and on the parameter $a$, which stands for the ratio of the natural line width $\Delta \lambda_{N}$ (including the effect of collisions) to the Doppler line width $\Delta \lambda_{\mathrm{D}}$

$a=\frac{\Delta \lambda_{N}}{\Delta \lambda_{\mathrm{D}}}$

According to the quantum theory of radiation (Heitler 1954), independently of the wavelength we have $\Delta \lambda_{N}=1.18 \times 10^{-4} \AA$, and the Doppler width is expressed by the known formula

$\Delta \lambda_{\mathrm{D}}=\frac{\lambda_{0}}{c} \sqrt{\frac{2 k T}{m}}$

where $\lambda_{0}$ is the wavelength of the spectral line, $c$ is speed of light, $k$ is the Boltzmann constant, $m$ is mass of the atom, and $T$ is the (kinetic) temperature. According to the different estimates (Ceplecha 1964, 1965, 1967, 1971; Harvey 1973a; Nagasawa 1978; Babadzhanov \& Getman 1980; Bronshten \& Kokhirova 1989; Borovička 1993), the excitation temperature $T$ of a meteor coma lies in the range within $1500 \mathrm{~K}-4400 \mathrm{~K}$. A possible cause of this spread consists in the choice of spectral lines of different elements from which the excitation temperatures $T$ were determined. In the range $1500 \mathrm{~K}-4400 \mathrm{~K}$, of which the mean value is $T=3000 \mathrm{~K}$, the parameter $a$ is not in excess of $a=0.01$ for the $\mathrm{H}$ and $\mathrm{K}$ lines of ionized calcium. So, as in the case of the majority of stellar spectra, the condition $a \ll 1$ is realized. The broadening of the CaII lines is mainly the result of the Doppler effect, i.e. it is owing to thermal motions of CaII ions and electrons, and of collisions between them. Note that from the emission curve of growth constructed for the FeI multiplets, Ceplecha (1964) also found $a=0.01$. Plotting the point corresponding to ionized calcium on the FeI emission curve of growth shows that this point lies in the right part of the curve of growth, where self-absorption is clearly present particularly well.

Investigating the absorption volume in the presence of both natural damping and the Doppler effect (i.e. $a \neq 0$ ), Held (1931) compiled tables of the values $B$, the total intensity of an absorption in a line, for different values of $f \lambda N / 2 \pi \xi_{0}$ and for $a=0 ; 0.001 ; 0.01 ; 0.1 ; 1.0$. Here $f$ is the oscillator strength, $N$ is the particle concentration, and $\xi_{0}$ is the thermal velocity of the particles. The values of $f \lambda N / 2 \pi \xi_{0}$ in Held's tables are given for the $\mathrm{K}$ line of CaII. Corresponding values for the $\mathrm{H}$ line of CaII may be found from the relationship

$\left(\frac{f \lambda N_{\text {CaII }}}{2 \pi \xi_{\text {CaII }}}\right)_{\mathrm{H}} /\left(\frac{f \lambda N_{\text {CaII }}}{2 \pi \xi_{\text {CaII }}}\right)_{\mathrm{K}}=\frac{f_{\mathrm{H}} \lambda_{\mathrm{H}}}{f_{\mathrm{K}} \lambda_{\mathrm{K}}}=0.504$

where $N_{\text {CaII }}$ is the calcium ion column concentration; $f_{\mathrm{H}}$ and $f_{\mathrm{K}}$ are the oscillator strengths of the $\mathrm{H}$ and $\mathrm{K}$ lines of CaII; $\lambda_{\mathrm{H}}=$ $3968.47 \AA$ and $\lambda_{\mathrm{K}}=3933.66 \AA$ are the wavelengths of those lines respectively; and $\xi_{\text {CaII }}$ is the thermal velocity of calcium ions.

Choosing the values of $B / 2 \Delta \lambda_{\mathrm{D}}$ according to the values of $f \lambda N / 2 \pi \xi_{0}$ from Held's tables when $a=0.01$, we express the ratio of these absorption intensities for the $\mathrm{H}$ and $\mathrm{K}$ lines of CaII through the observed values of emission intensities $I_{\mathrm{K}}$ and $I_{\mathrm{H}}$ of these lines:

$\frac{\left(B / 2 \Delta \lambda_{\mathrm{D}}\right)_{\mathrm{K}}}{\left(B / 2 \Delta \lambda_{\mathrm{D}}\right)_{\mathrm{H}}}=\frac{\lambda_{\mathrm{H}}}{\lambda_{\mathrm{K}}} \frac{I_{\mathrm{K}}}{I_{\mathrm{H}}}$

or

$\frac{I_{\mathrm{K}}}{I_{\mathrm{H}}}=\frac{\lambda_{\mathrm{K}}}{\lambda_{\mathrm{H}}} \frac{\left(B / 2 \Delta \lambda_{\mathrm{D}}\right)_{\mathrm{K}}}{\left(B / 2 \Delta \lambda_{\mathrm{D}}\right)_{\mathrm{H}}}$

From Eq. (5) we obtain the set of values $I_{\mathrm{K}} / I_{\mathrm{H}}$ for the selected intensities of absorption $B$. Here we take into account that the emission intensity $I$ in a spectral line corresponds to the absorption intensity $B$. Further, it is possible to calculate the values $(N / \xi)_{\text {CaII }}$ corresponding to the values $f \lambda N / 2 \pi \xi_{0}$ taken from Held's tables. So, for each value of $\log (N / \xi)_{\text {CaII }}$ the corresponding value $I_{\mathrm{K}} / I_{\mathrm{H}}$ is determined. This dependence is given in Fig. 1 (Viazanitsin 1947).

Using the equality (Viazanitsin 1947)

$\frac{B}{2 \Delta \lambda_{\mathrm{D}}}=\frac{c I}{2 \lambda J \xi_{\text {CaII }}}$

and rewriting it in the form (Viazanitsin 1947)

$J \xi_{\text {CaII }}=\left[\frac{2 c / 2 \lambda}{B / \Delta \lambda_{\mathrm{D}}}\right] I$, 


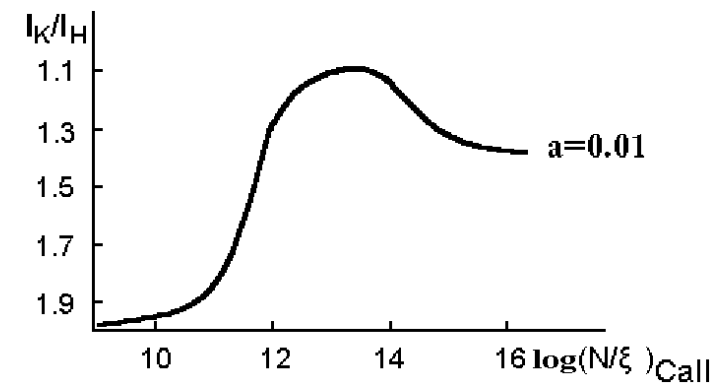

Fig. 1. Dependence of the ratio $I_{\mathrm{K}} / I_{\mathrm{H}}$ on $\log (N / \xi)_{\text {CaII }}$ when $a=0.01$.

and then designating $2 c / 2 \lambda /\left(B / \Delta \lambda_{\mathrm{D}}\right)$ through $D_{\mathrm{H}}$ for the $\mathrm{H}$ line we get:

$J \xi_{\text {CaII }}=D_{\mathrm{H}} I_{\mathrm{H}}$,

where $I$ is the radiation intensity of ionized calcium lines, and $J$ is the intensity of the exciting radiation of these lines (both values are in erg $\mathrm{cm}^{-2} \mathrm{~s}^{-1} \mathrm{ster}^{-1}$ ). Since the values $B / 2 \Delta \lambda_{\mathrm{D}}$ are a function of $(N / \xi)_{\text {CaII }}$, the values $D_{\mathrm{H}}$ also depend upon $(N / \xi)_{\text {CaII }}$. From the dependence $D_{\mathrm{H}}$ on $\log (N / \xi)_{\text {CaII }}$ (when $a=0.01$ ) obtained by Viazanitsin and presented in Fig. 2 it is possible to find the values $D_{\mathrm{H}}$ according to known values of $\log (N / \xi)_{\text {CaII }}$. Multiplying $D_{\mathrm{H}}$ by $I_{\mathrm{H}}$ we find $J \xi_{\text {CaII }}$. The calcium thermal velocity calculated for the excitation temperature $T=3000 \mathrm{~K}$ is equal to $\xi_{\text {CaII }}=1.11 \mathrm{~km} \mathrm{~s}^{-1}$ (Ceplecha 1964), which is very close to the value obtained by Viazanitsin (1947) from the solar prominence spectra. Dividing $J \xi_{\text {CaII }}$ by this value of the thermal velocity we obtain the intensity of the exciting radiation $J$. From the latter we determine the corresponding temperature $T$ from the Planck formula

$J=\frac{2 \pi c^{2} h}{\lambda^{5}} \frac{1}{\exp (h c / \lambda k T-1)}$,

where $h$ is the Planck constant, and $\lambda$ is the wavelength of ionized calcium lines. We used the values of all constants from Allen (1973).

For the determination of the calcium ion concentration and of the excitation temperature in meteor comae the prism spectra of eight meteors were available; they were photographed in variuos years by the spectral aggregate of the meteor patrol in the Gissar observatory of the Institute of Astrophysics, Tajik Academy of Sciences. This aggregate consists of 6 small cameras (the focal ratio $D / F=1: 2.5$ and focal length $F=250 \mathrm{~mm}$ ) equipped with objective prisms of refracting angle $25^{\circ}$. In the spectra the doublet lines of ionized calcium were identified. The results of the determination of the column concentration of calcium ions for two of these spectra were published earlier (Bronshten \& Kokhirova 1989).

The basic data on the atmospheric trajectories of the meteors studied were published by Babadzhanov et al. (1966, 1968, 1982, 1998) and are summarized in Table 1, where the following data are given: $N$ is the number of the meteor, $L_{\odot}$ is the longitude of the Sun, $\alpha_{R}, \delta_{R}$ are geocentric right ascension and declination of the radiant, $V_{\infty}$ is preatmospheric velocity, $Z_{R}$ is zenith distance of the radiant, $H_{\mathrm{b}}, H_{\mathrm{m}}, H_{\mathrm{e}}$ are heights of beginning, maximum light and end respectively, $M_{\max }$ is absolute

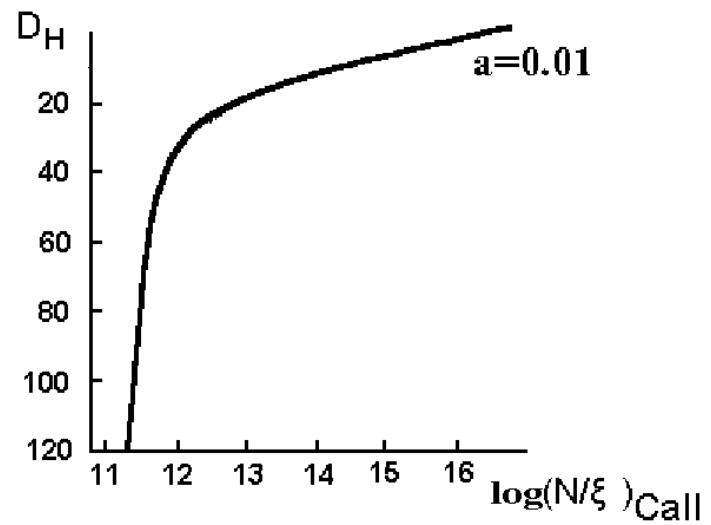

Fig. 2. Dependence of the values $D_{\mathrm{H}}$ on $\log (N / \xi)_{\text {CaII }}$ when $a=0.01$.

magnitude of the meteor at the point of maximum light, and membership of the meteor of known showers.

The intensities of the $\mathrm{H}$ and $\mathrm{K}$ lines of CaII were measured along the path of the meteors by the microdensitometer MD-100. For the photometric calibration we used the spectra of the stars in the vicinity of each meteor spectrum. The photographic densities of the red and blue parts of the spectra of the comparison stars were measured, and two photographic characteristic curves were constructed, which were then averaged. By means of these characteristic curves the photographic density scale of the spectrograms was converted to the magnitude scale, and then to the intensity scale. To scale the spectral sensitivity of emission lines, the spectrograms of early-type stars (e.g. $\alpha$ Lyrae, $\alpha$ Aquila etc.) with known spectral energy distribution were used as comparison spectra for each meteor spectrum separately. For the determination of the intensity of a spectral line of wavelength $\lambda$ the following correction to the values of its photographic magnitude was taken into account:

$\Delta m_{s}=-2.5 \log \left(\frac{E_{\lambda} \Delta \lambda}{E_{0} \Delta \lambda_{0}}\right)$,

where $E_{\lambda}$, the radiation energy of wavelength $\lambda$ of the comparison star (in erg $\mathrm{cm}^{-2} \mathrm{~s}^{-1} \mathrm{~cm}^{-1}$ ), was taken from the catalogue of Kharitonov et al. (1978); $\Delta \lambda$ is the wavelength interval contained in the slot width of the measuring instrument; $E_{0}=0.38 \mathrm{erg} \mathrm{cm}^{-2} \mathrm{~s}^{-1}$ is the radiation energy corresponding to $\lambda_{0}=5575 \AA$.

For the determination of the absolute magnitude $M$ of the $\mathrm{H}$ and $\mathrm{K}$ ionized calcium lines, the corrections for the standard distance of $100 \mathrm{~km}$, the zenith distance and the difference between the trailing velocities of meteor and stars (i.e. the Schwarzschild effect) were taken into account. The intensity $I$ (in erg s${ }^{-1}$ ) of a spectral line along a meteor path was found by the formula

$\log I=9.72-0.4 M$

Thus for eight meteor spectra the monochromatic light curves of the $\mathrm{H}$ and $\mathrm{K}$ lines of CaII were constructed and for selected points along the meteor trajectories the ratios $I_{\mathrm{K}} / I_{\mathrm{H}}$ were determined. As an example, in Figs. 3a,b these monochromatic light curves are shown for meteors 641623 and 830633. 
Table 1. Basic data on the atmospheric trajectory of meteors.

\begin{tabular}{lllllllllll}
\hline \hline$N$ & $\begin{array}{l}L_{\odot} \\
N\end{array}$ & $\alpha_{R}$ & $\delta_{R}$ & $V_{\infty}$ & $\cos Z_{R}$ & $H_{\mathrm{b}}$ & $H_{\mathrm{m}}$ & $H_{\mathrm{e}}$ & $M_{\max }$ & Shower \\
& 2000.0 & 2000.0 & 2000.0 & $\mathrm{~km} \mathrm{~s}^{-1}$ & & $\mathrm{~km}$ & $\mathrm{~km}$ & $\mathrm{~km}$ & & \\
\hline 600361 & $208^{\circ} .0$ & 91.3 & 14.5 & 74.9 & 0.543 & 127.3 & 100.9 & 93.6 & -11.9 & Orionid \\
641623 & $136^{\circ} .6$ & $284^{\circ} .5$ & $45^{\circ} .7$ & 24.6 & 0.986 & 94.6 & 80.5 & 77.8 & -8.1 & Sporad. \\
641872 & 140.5 & $282^{\circ} .0$ & $46^{\circ} .4$ & 24.6 & 0.967 & 104.3 & 85.0 & 74.5 & -12.0 & Sporad. \\
690803 & 140.7 & $47^{\circ} .2$ & $56^{\circ} .7$ & 60.0 & 0.507 & 110.0 & 92.0 & 87.5 & $<-12.6$ & Perseid \\
830633 & $136^{\circ} .6$ & $44^{\circ} .3$ & $59^{\circ} .1$ & 58.7 & 0.769 & 111.4 & 92.3 & 81.8 & -5.5 & Perseid \\
830811 & 139.4 & $45^{\circ} .4$ & $58^{\circ} .0$ & 59.5 & 0.811 & 108.5 & 91.5 & 88.1 & $<-6.5$ & Perseid \\
830953 & 141.4 & $342^{\circ} .4$ & -18.3 & 30.4 & 0.576 & - & - & 57.8 & $<-9.3$ & Sporad. \\
900315 & $127^{\circ} .3$ & $45^{\circ} .9$ & $56^{\circ} .2$ & 60.0 & 0.507 & 110.0 & 91.1 & 87.3 & $<-6.5$ & Perseid \\
\hline
\end{tabular}

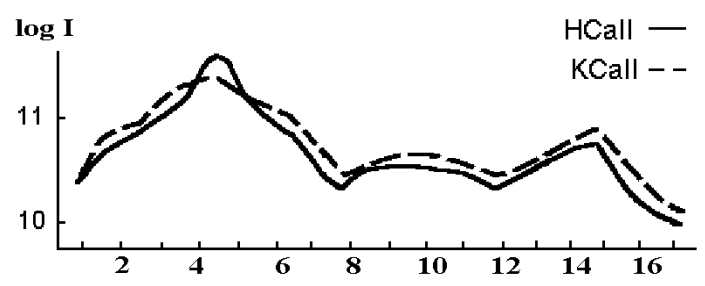

(a)

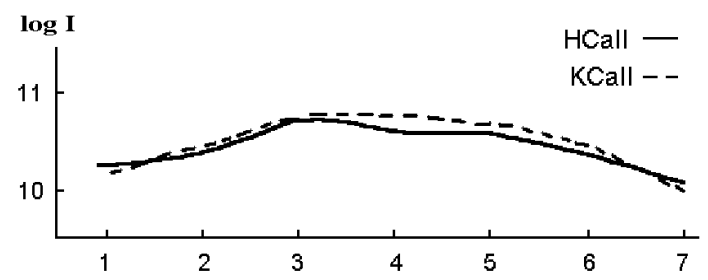

(b)

Fig. 3. a) Monochromatic light curves for the meteor 641623 . b) Monochromatic light curves for the meteor 830633 .

For the points mentioned above, from the graph of Fig. 1 we found $\log (N / \xi)_{\text {CaII }}$. Taking into account the conclusion proven above of the importance of the Doppler effect for the broadening of ionized calcium lines, and proceed from the shape of the curve of growth when $a=0.01$, the selection of the values $I_{\mathrm{K}} / I_{\mathrm{H}}<1.4$ was made from the left branch of the graph in Fig. 1 .

The results of the determination of the calcium ion column concentration $\log (N)_{\text {CaII }}$ when $\xi_{\text {CaII }}=1.11 \mathrm{~km} \mathrm{~s}^{-1}$, are given for each meteor spectrum in Tables 2-9. In order to transform the column density $N_{\text {CaII }}$ into the number (i.e. volume) density $n_{\text {CaII }}$ we used the following relation (Borovička 1993):

$n_{\text {CaII }}=\frac{N_{\text {CaII }}}{s}$,

where $s$ is the geometrical thickness of the meteor along the line of sight:

$s=\frac{b}{\sin \alpha}$.

Here $b$ is the width of meteor trail measured by us from the photographs obtained by the method of instantaneous exposure using the long-focus cameras MK-75 $(D / F=1: 3.5, F=$ $750 \mathrm{~mm}$ ), and $\alpha$ is the angle between the line of sight and the direction of meteor flight determined from the known meteor trajectory in space. The determined values of $b$ in meters and of $n_{\text {CaII }}$ are given also in Tables 2-9. Here when $(x)$ is given in the column heading, the values in the column are to be multiplied by $10^{x}$. $N_{\text {CaII }}$ is given in $\mathrm{cm}^{-2}$ and $n_{\text {CaII }}$ is given in $\mathrm{cm}^{-3}$.

As is seen from Tables 2-9, the ion concentrations $n_{\text {CaII }}$ of these meteors are in the range within $10^{13}-10^{15} \mathrm{~cm}^{-3}$, which is in satisfactory agreement with the values obtained by other methods. For example, using the ECG method Ceplecha (1964) found a value $n_{\text {CaII }}=10^{15} \mathrm{~cm}^{-3}$ for his fireball spectrum.

Analysing the results of the determination of the calcium ion concentration along each meteor trajectory by means of comparison with the corresponding monochromatic light curve and with the excitation temperature changes along the meteor trajectory (Tables 2-9), we come to the following conclusions. As a rule, the concentration of CaII ions has its minimum value on the section of the light curve corresponding to the maximum brightness and, consequently, to the maximum activity of evaporation process. This was also revealed earlier from treatment of two meteor spectra (Bronshten \& Kokhirova 1989). This shows that with the intensification of evaporation, both excitation and ionization weaken. Expansion of the meteor coma leads to a decrease of the ion concentration per unit of volume. This becomes particularly apparent for the meteors that shows flares in brightness, when at the sharp increase of the light intensity, the concentration of calcium ions decreases sharply. For meteors with more or less smooth light curves, i.e. for the meteors 830633 and 641872 , the variation of the calcium ion concentration also has a smooth character along the meteor path. However, here some reduction is observed of the ion concentration in the light curve segments with the highest intensity.

\section{Determination of the concentration of free electrons in a meteor coma}

To solve this problem it is necessary to make some simplified assumptions concerning meteor ionization. First of all, we assume local thermodynamic equilibrium in the meteor coma. Then, proceeding from the determined excitation temperature, 
which does not exceed $3000 \mathrm{~K}$ for the meteors under consideration, we may assume that only neutral and singly ionized atoms are present in the coma. Besides, in a meteor coma the total concentration of free electrons $n_{\mathrm{e}}$, which is responsible for the quasi-neutrality inside meteor trail as a whole, in reality is the sum of $n_{\mathrm{e}}$ from different elements identified in spectrum.

As was shown by Borovička (1993), the total electron density $n_{\mathrm{e}}$, under the condition of quasi-neutrality and using the simple geometrical model of the meteors, is determined by the following equation

$n_{\mathrm{e}}=\left(\sum_{j} n_{j} / \Omega_{j}(T)\right)^{1 / 2}$,

where $n_{j}$ is the density of neutral atoms of a given element, and $\Omega_{j}(T)$ is the function of the temperature for the same element. So, the total electron density $n_{\mathrm{e}}$ depends upon the sum $n_{j}$ of all elements.

The volume electron density (i.e. the concentration of free electrons) $n_{\mathrm{e}}$ may be estimated in the following manner. From the results of combined photographic and radar observations of meteors (Verniani \& Hawkins 1964; Babadzhanov 1969) the following dependence was obtained of the absolute magnitude $M$ of meteors on the initial line density $q$ of electrons in the reflecting segment of the meteor column

$M=34.3-2.5 \log q$.

Thus, using Eq. (12) the line electron density $q$ of a meteor can be determined from its absolute magnitude.

Assuming that because of the Coulomb attraction of positive ions almost all ions remain in the meteor column, the mean volume concentration of free electrons $n_{\mathrm{e}}$ in the meteor column may be obtained as (Nagasawa 1978)

$n_{\mathrm{e}}=\frac{q}{\pi r_{\mathrm{m}}^{2}}$,

where $r_{\mathrm{m}}$ is the mean radius of the meteor column. Though the mean radius of the meteor column is larger for heavier elements, it is difficult to estimate these differences in the real column. Therefore for all elements we can assume the same emitting volume and radius $r_{\mathrm{m}}$ of the meteor column.

The mean radius $r_{\mathrm{m}}$ of the meteor column is related to the radiation time in the following form (Nagasawa 1978)

$\log (t / l)=0.237\left(r_{\mathrm{m}} / l\right)-4.251-0.234 /\left(r_{\mathrm{m}} / l\right)$,

where $t$ is the duration of the meteor radiation in seconds, and $l$ is the mean free path of a meteor particle in meters. The value of $l$ may be calculated from

$l=\frac{1}{\pi\left(\sigma_{1}+\sigma_{2}\right)^{2} n_{a}}$,

where $\sigma_{1}, \sigma_{2}$ are the radii of meteor particles and air molecules respectively, $n_{a}$ is the density of air molecules in $\mathrm{cm}^{-3}$ given in the US Standard Atmosphere (1962), which for the height range between $90 \mathrm{~km}$ and $100 \mathrm{~km}$ is approximated as

$n_{a}=1.27347 \times 10^{21} \exp (-0.18659 h)$, where $h$ is the height in $\mathrm{km}$. Equation (15) for $l$ is valid only in the case where the speeds of meteor particles are far greater than that of air molecules. For the atoms of $\mathrm{CaI}$ we have $\sigma_{1}+$ $\sigma_{2}=3.84 \AA$ and $t=0.032 \mathrm{~s}$ (Nagasawa 1978).

Using Eq. (16) and taking into account that the height of maximum brightness of CaI (4226) in the spectrum of meteor 690803 is equal to $h=92 \mathrm{~km}$, we find $n_{a}=4.46 \times$ $10^{13} \mathrm{~cm}^{-3}$. Further by the formula (15) we calculated the mean free path of the calcium atoms to be equal to $l_{\mathrm{CaI}}=4.84 \mathrm{~cm}$. Substituting the obtained values of $n_{a}$ and $l_{\mathrm{CaI}}$ into Eq. (14), we find $r_{\mathrm{m}}=42 \mathrm{~cm}$, which is in accordance with the estimates of Nagasawa (1978), who concluded that $r_{\mathrm{m}}$ does not exceed $0.5 \mathrm{~m}$.

The absolute magnitude of meteor 690803 according to its monochromatic light curve is $M(\mathrm{CaI})=-8 \mathrm{~m}$. If we substitute this into Eq. (12) we derive for the meteor line electron density the value $q=2.3 \times 10^{17} \mathrm{~cm}^{-1}$. Finally, from Eq. (13) we obtain $n_{\mathrm{e}}=4.1 \times 10^{13} \mathrm{~cm}^{-3}$.

In the spectrum of meteor 690803 the spectral lines of neutral MgI (5175) and single ionized MgII (4481) were also available for measurements. From similar calculations for these lines, we obtained $n_{\mathrm{e}}=3.7 \times 10^{13} \mathrm{~cm}^{-3}$. It turned out that the values of the electron volume density at the segments of maximum brightness of the monochromatic light curves derived for meteor 690803 from the lines of magnesium and calcium are close to each other. The values of $n_{\mathrm{e}}$ calculated by us are in good agreement with the results obtained by other researchers. So, by the method used in the present paper, Nagasawa (1978) for two Leonid spectra estimated the electron volume density in a meteor coma as $n_{\mathrm{e}} \sim 10^{12} \mathrm{~cm}^{-3}$. From the spectrum of the fireball EN151068 Borovička (1993) obtained values for $n_{\mathrm{e}}$ equal to $10^{12} \mathrm{~cm}^{-3}$ at the beginning of the meteor path and $10^{13} \mathrm{~cm}^{-3}$ at the segments of the maximum of the light curve.

For meteor 690803 at the point of maximum brightness the value of the electron concentration is $n_{\mathrm{e}}=4.1 \times 10^{13} \mathrm{~cm}^{-3}$, which is in satisfactory agreement with the calcium ion concentration $n_{\text {CaII }}=7.9 \times 10^{13} \mathrm{~cm}^{-3}$. This supports the suggestion that the CaII ions are the main source of free electrons in the meteor coma (see e.g. Bronshten 1981). It also confirms the conclusions of Borovička \& Betlem (1997) that 98.5-99.3 percent of the neutral $\mathrm{CaI}$ atoms are ionized along the whole meteor trajectory.

If the values of $n_{\text {CaII }}$ and $n_{\mathrm{e}}$ are known, then it is possible to define the concentration of neutral calcium atoms $n_{\text {CaI }}$. The ratio of the number of calcium ions $n_{\text {CaII }}$ to the number of neutral calcium atoms $n_{\text {CaI }}$ may be determined by Saha's ionization formula in the following form

$\log \left(\frac{n_{\mathrm{CaII}}}{n_{\mathrm{CaI}}} n_{\mathrm{e}}\right)=-\chi_{\gamma, \gamma+1} \theta \frac{3}{2} \log \theta+20.9366+\log \left(\frac{2 U_{\gamma+1}}{U_{\gamma}}\right)$,

where $n_{\mathrm{e}}$ is the number of free electrons per $\mathrm{cm}^{-3}, \chi_{\gamma, \gamma+1}=3.1$ is the ionization potential of an atom from ionization stage $\gamma$ to $\gamma+1$, in eV, $\theta=5040 / T$ is the characteristic temperature, $U_{\gamma}$ and $U_{\gamma+1}$ are partition functions or effective statistical weights of neutral atom and singly ionized atom, respectively, under the given conditions of excitation. In our case the temperature 
of ionization is close to $\theta=1$ and therefore we used (Allen 1973): $U_{\gamma}=3.55$ and $U_{\gamma+1}=3.47$. This possibility was available only for the spectrum of meteor 690803 . Thus the value of $n_{\mathrm{CaI}}=2.45 \times 10^{7} \mathrm{~cm}^{-3}$ obtained by us at the maximum light point is close to the values of $n_{\mathrm{CaI}}$ calculated by Borovička (1993) for the spectrum of the fireball EN151068 at different points along the trajectory.

Examination of the available results of quantitative analysis of meteor spectra allows us to conclude that, probably, such physical parameters of meteor comae as the electron concentration and, in particular, the ion concentration of calcium and magnesium, do not depend on the orbital elements and atmospheric trajectories of meteors, but, first of all, depend on the parameters characterizing the excitation and ionization conditions, namely on the excitation and ionization temperature, and further on the electron temperature which in turn depend on the meteoroid composition.

\section{Conclusions}

1. For the first time in quantitative analysis of meteor spectra a method is adopted, that was examinated long ago in the investigations of the solar prominences. Use of this method for quantitative analysis of meteor spectra overcomes one of the main difficulties concerning the accounting the selfabsorption, which is especially strong for the ionized calcium spectral lines. Another important advantage of this method is that it can be used not only for the grating, but also for prism spectra. The method is sufficiently simple, it does not require complicated mathematical methods. The results obtained by its application, and comparison of these results with those obtained by other methods prove its reliability and validity.

On the basis of this method, prism spectra of eight meteors belonging to Perseids and Orionids, and to sporadic background have been studied.

2. The meteor coma radiation in the $\mathrm{H}$ and $\mathrm{K}$ lines of ionized calcium has been investigated along the meteor trajectories. The excitation temperature, the calcium ion concentration and their variation as a function of meteor height are calculated. For two spectra the electron concentration has also been calculated for the meteor trail segments corresponding to the maximum of brightness.

In the segments of maximum intensity of the meteor monochromatic light curves the derived excitation temperature $T$ is within 1500-2000 K, which is in satisfactory agreement with results obtained by other methods (Ceplecha 1964, 1965, 1967, 1971; Nagasawa 1978; Harvey 1973a; Babadzhanov \& Getman 1980).

Variation of the temperature with height is similar to the form of the monochromatic light curve, i.e. it grows gradually with the rise of intensity for the meteors that have smooth light curves. The temperature had a maximum value at the flare, and fell at the end of meteor trajectories.

The values of the calcium ion concentration determined by the new method of meteor spectroscopy are in the range $\sim 10^{13}-10^{15} \mathrm{~cm}^{-3}$. We may conclude that the calcium abundance in meteoroids is defined only by the their composition. According to the abundance of calcium ions the meteoroids studied in this paper may be related to chondrites or carbonaceous chondrites (Ceplecha 1964; Millman 1972; Nagasawa 1978; Borovička 1993; Borovička \& Betlem 1997).

Behavior of the calcium ion concentration along the meteor trajectories differs essentially for meteors with and without flares. Such dependency of the calcium ion concentration upon temperature jumps shows the strong dependency of ionization processes on the temperature.

As is seen from a comparison of the functions $T(h)$ and $\log n_{\text {CaII }}(h)$ for meteors without flares, with the gradual increasing of the temperature the $\log n_{\text {CaII }}$ is increasing too, and no jump of the calcium ion concentration is observed along the meteor path. This confirms a suggestion that the meteor coma radiation approaches at most to the thermal equilibrium state for meteors without flares.

For meteors with flares, the calcium ion concentration is rising before the sharp increase in the temperature, but it falls sharply at the moment of the flare. This confirms that the values of some physical characteristics of meteor coma fall at the point of the sudden temperature change. For example, the calcium ion concentration is decreasing because of the accelerated ionization in the flare and, as consequence, of the acceleration of the expansion of the meteor coma. The same effect was found for the absolute abundance of iron in the radiating meteor coma.

The values obtained here of the concentration of free electrons $n_{\mathrm{e}}$ at the point of maximum light of the meteor coma, equal to $\sim 10^{13}-10^{14} \mathrm{~cm}^{-3}$, prove unquestionably the fact that almost 99 percent of calcium is in the ionized state and, consequently, calcium is one of the main sources of free electrons in a meteor coma.

Acknowledgements. The authors would like to express their gratitude to the anonymous reviewer for useful comments which significantly improved the paper. The annotation of the English copy editor of the A\&A Dr. Jet Katgert is also appreciated.

\section{References}

Allen, C. W. 1973, Astrophysical Quantities, 3rd ed., University of London (The Althone Press)

Babadzhanov, P. B., Suslova, N. N., \& Karaselnikova, S. A. 1966, Bull. Inst. Astrophys., Tajik Acad. Sci., 41-42, 3

Babadzhanov, P. B., Getman, T. I., Zausaev, A. F., et al. 1968, Bull. Inst. Astrophys., Tajik Acad. Sci., 49, 3

Babadzhanov, P. B. 1969, Doklady Akademii Nauk SSSR, 184,4

Babadzhanov, P. B., \& Getman, V. S. 1980, Doklady Akademii Nauk Tajik. SSR, 13, 12

Babadzhanov, P. B., Getman, T. I., Konovalova, N. A., et al. 1982, Bull. Inst. Astrophys., Tajik Acad. Sci., 73, 22

Babadzhanov, P. B., Zubareva, T. I., Konovalova, N. A., et al. 1998, Bull. Inst. Astrophys., Tajik Acad. Sci., 82, 16

Blajko, S. N. 1932, AJ, 9, 3-4, 146

Borovička, J. 1993, A\&A, 279, 627

Borovička, J., \& Betlem, H. 1997, Planet. Space Sci., 45, 5, 563

Bronshten, V. A. 1981, Fizika meteornykh yavlenij, Nauka, Moscow; English translation: Physics of Meteoric Phenomena (Dordrecht: Reidel) (1983) 
Bronshten, V. A., \& Kokhirova, G. I. 1989, Doklady Akademii Nauk Tajik. SSR, 32, 4, 234

Ceplecha, Z., \& Rajchl, J. 1963, Bull. Astron. Inst. Czechosl., 14, 2, 29

Ceplecha, Z. 1964, Bull. Astron. Inst. Czechosl., 15, 3, 102

Ceplecha, Z. 1965, Bull. Astron. Inst. Czechosl., 16, 2, 88

Ceplecha, Z. 1967, Bull. Astron. Inst. Czechosl., 18, 303

Ceplecha, Z. 1971, Bull. Astron. Inst. Czechosl., 22, 5, 219

Cook, A. F., Halliday, I., \& Millman, P. M. 1971, Can. J. Phys., 49, 1738

Halliday, I. 1961, Publ. Dominion Obs. Ottawa, 25(1), 3

Halliday, I. 1987, A\&A, 187, 921

Harvey, G. A. 1971, AJ, 165, 3, 669

Harvey, G. A. 1973a, In Evolutionary and physical properties of meteoroids, ed. C. L. Hemenway, P. M. Millman, \& A. F. Cook, NASA-SP, 319, 103

Harvey, G. A. 1973b, J. Geophys. Res., 78, 3913

Heitler, W. 1954, The quantum theory of radiation, 3rd ed. (Oxford: Clarendon Press)
Held, V. D. 1931, Zs.f. Phys., 70, 508

Kharitonov, A. V., Tereshchenko, V. M., \& Knyazeva, L. N. 1978, Svodnyij spektrofotometricheskij katalog zvezd (Alma-Ata: Nauka), 197

Millman, P. M. 1933, Pop. Astron., 41, 6

Millman, P. M. 1935, Ann. Harvard Coll. Obs., 82, 7, 149

Millman, P. M., \& Halliday, I. 1961, Planet. Space Sci., 5, 137

Millman, P. M., Cook, A. F., \& Hemenway, C. L. 1971, Can. J. Phys., 49, 1365

Millman, P. M. 1972, J.R. Astron. Soc. Canada, 66, 201

Nagasawa, K. 1978, Ann. Tokyo Astron. Obs. 2nd Ser., 16, 4, 157

Rajchl, J. 1961, Bull. Astron. Inst. Czechosl., 12, 4, 29

Rajchl, J. 1974, Bull. Astron. Inst. Czechosl., 25, 1, 34

Verniani, F., \& Hawkins, G. S. 1964, AJ, 140, 4, 1590

Viazanitsin, V. P. 1947, Izvest. GAO Poulkovo, 17, 136, 1

US Standard Atmosphere 1962 (Washington DC: US Government Printing Office) 
P. B. Babadzhanov and G. I. Kokhirova: Calcium ions in a meteor coma, Online Material p 1

\section{Online Material}


P. B. Babadzhanov and G. I. Kokhirova: Calcium ions in a meteor coma, Online Material p 2

Table 2. Concentration of the calcium ions in the coma of meteor 600361 .

\begin{tabular}{|c|c|c|c|c|c|c|c|c|c|c|}
\hline Points & $I_{\mathrm{K}} / I_{\mathrm{H}}$ & $\begin{array}{l}I_{\mathrm{H}} \\
(12) \\
\end{array}$ & $\log (N / \xi)_{\text {CaII }}$ & $D_{\mathrm{H}}$ & $\begin{array}{l}J \xi_{\text {CaII }} \\
(14) \\
\end{array}$ & $\begin{array}{l}J \\
(9) \\
\end{array}$ & $T$ & $\log N_{\text {CaII }}$ & $\begin{array}{l}b \\
\mathrm{~m}\end{array}$ & $\log n_{\text {CaII }}$ \\
\hline 1 & 1.20 & 0.3 & 12.4 & 27 & 0.08 & 0.06 & 1542 & 17.4 & 4.8 & 14.7 \\
\hline 2 & 1.15 & 1.2 & 12.7 & 24 & 0.29 & 0.20 & 1629 & 17.7 & 5.2 & 14.9 \\
\hline 3 & 1.44 & 2.6 & 11.8 & 44 & 1.10 & 0.82 & 1737 & 16.8 & 5.7 & 14.0 \\
\hline 4 & 1.20 & 3.0 & 12.4 & 27 & 0.81 & 0.58 & 1708 & 17.4 & 6.1 & 14.6 \\
\hline 5 & 1.66 & 5.4 & 11.4 & 94 & 5.1 & 3.6 & 1870 & 16.4 & 6.6 & 13.6 \\
\hline 6 & 1.66 & 5.4 & 11.4 & 94 & 5.1 & 3.6 & 1870 & 16.4 & 7.1 & 13.5 \\
\hline 7 & 1.74 & 4.3 & 11.3 & 110 & 4.7 & 3.4 & 1863 & 16.3 & 7.1 & 13.4 \\
\hline 8 & 1.59 & 31.0 & 11.6 & 70 & 2.2 & 1.6 & 1792 & 16.6 & 7.2 & 13.7 \\
\hline 9 & 1.51 & 3.0 & 11.7 & 54 & 1.6 & 1.1 & 1765 & 16.7 & 7.8 & 13.8 \\
\hline 10 & 1.44 & 2.6 & 11.8 & 44 & 1.1 & 0.82 & 1737 & 16.8 & 8.5 & 13.9 \\
\hline 11 & 1.59 & 1.7 & 11.6 & 70 & 1.2 & 0.86 & 1741 & 16.6 & 8.5 & 13.7 \\
\hline 12 & 1.14 & 1.3 & 12.8 & 23 & 0.30 & 0.21 & 1633 & 17.8 & 8.5 & 14.9 \\
\hline 13 & 1.26 & 0.54 & 12.1 & 31 & 0.17 & 0.12 & 1591 & 17.2 & 8.1 & 14.3 \\
\hline
\end{tabular}

Table 3. Concentration of the calcium ions in the coma of meteor 641623.

\begin{tabular}{ccccccccccc}
\hline \hline \multirow{2}{*}{ Points } & $I_{\mathrm{K}} / I_{\mathrm{H}}$ & $I_{\mathrm{H}}$ & \multirow{2}{*}{$\log (N / \xi)_{\text {CaII }} D_{\mathrm{H}}$} & $J \xi_{\text {CaII }} J$ & $T$ & $\log N_{\text {CaII }} b$ & $\log n_{\text {CaII }}$ \\
\multicolumn{2}{c}{} & $(10)$ & & & $(12)$ & $(6)$ & & & $\mathrm{m}$ & \\
\hline 2 & 1.51 & 5.0 & 11.7 & 52 & 2.6 & 18.0 & 1471 & 16.7 & 17.0 & 13.5 \\
3 & 1.26 & 7.2 & 12.1 & 32 & 2.3 & 16.0 & 1464 & 17.2 & 22.0 & 13.8 \\
4 & 1.38 & 16.0 & 11.9 & 38 & 6.0 & 43.0 & 1522 & 16.9 & 34.0 & 13.3 \\
7 & 1.32 & 8.2 & 12.0 & 35 & 2.9 & 21.0 & 1477 & 17.0 & 29.0 & 13.4 \\
8 & 1.51 & 3.8 & 11.7 & 52 & 2.0 & 14.0 & 1455 & 16.7 & 26.0 & 13.2 \\
9 & 1.32 & 2.4 & 12.0 & 36 & 0.9 & 6.1 & 1408 & 17.0 & 24.0 & 13.6 \\
10 & 1.15 & 3.6 & 12.7 & 24 & 0.9 & 6.2 & 1409 & 17.7 & 22.0 & 14.3 \\
11 & 1.26 & 3.8 & 12.1 & 32 & 1.2 & 8.6 & 1427 & 17.2 & 19.0 & 13.9 \\
12 & 1.26 & 3.3 & 12.1 & 32 & 1.0 & 7.5 & 1419 & 17.2 & 16.0 & 14.0 \\
13 & 1.26 & 2.5 & 12.1 & 32 & 0.8 & 5.7 & 1404 & 17.2 & 15.0 & 14.0 \\
14 & 1.26 & 4.1 & 12.1 & 32 & 1.3 & 9.4 & 1432 & 17.2 & 23.0 & 13.8 \\
15 & 1.38 & 6.5 & 11.9 & 38 & 2.5 & 1.8 & 1344 & 16.9 & 31.0 & 13.4 \\
17 & 1.44 & 1.1 & 11.8 & 42 & 0.46 & 3.3 & 1375 & 16.8 & 13.0 & 13.6 \\
\hline
\end{tabular}


P. B. Babadzhanov and G. I. Kokhirova: Calcium ions in a meteor coma, Online Material p 3

Table 4. Concentration of the calcium ions in the coma of meteor 641872.

\begin{tabular}{|c|c|c|c|c|c|c|c|c|c|c|}
\hline Points & $I_{\mathrm{K}} / I_{\mathrm{H}}$ & $\begin{array}{l}I_{\mathrm{H}} \\
(12)\end{array}$ & $\log (N / \xi)_{\text {CaII }}$ & $D_{\mathrm{H}}$ & $\begin{array}{l}J \xi_{\text {CaII }} \\
(14)\end{array}$ & $\begin{array}{l}J \\
(9)\end{array}$ & $T$ & $\log N_{\text {CaII }}$ & $\begin{array}{l}b \\
\mathrm{~m}\end{array}$ & $\log n_{\text {CaII }}$ \\
\hline 2 & 1.74 & 0.9 & 11.3 & 120 & 1.1 & 0.8 & 1733 & 16.3 & 20.0 & 13.0 \\
\hline 3 & 1.35 & 3.2 & 11.9 & 38 & 1.2 & 0.9 & 1741 & 16.9 & 19.0 & 13.6 \\
\hline 4 & 1.95 & 4.2 & 10.3 & 230 & 9.7 & 6.9 & 1933 & 15.3 & 24.0 & 11.9 \\
\hline 5 & 1.51 & 6.1 & 11.7 & 52 & 3.2 & 2.3 & 1825 & 16.7 & 20.0 & 13.3 \\
\hline 6 & 1.37 & 6.7 & 11.9 & 38 & 2.5 & 1.8 & 1805 & 16.9 & 19.0 & 13.6 \\
\hline 7 & 1.37 & 6.7 & 11.9 & 38 & 2.5 & 1.8 & 1805 & 16.9 & 19.0 & 13.7 \\
\hline 8 & 1.46 & 6.1 & 11.8 & 44 & 2.7 & 1.9 & 1810 & 16.8 & 19.0 & 13.5 \\
\hline 9 & 1.59 & 3.7 & 11.6 & 64 & 2.4 & 1.7 & 1799 & 16.6 & 19.0 & 13.3 \\
\hline 10 & 1.92 & 1.2 & 10.6 & 214 & 2.6 & 1.8 & 1806 & 15.6 & 27.0 & 12.2 \\
\hline 11 & 1.61 & 0.5 & 11.6 & 64 & 0.3 & 0.2 & 1631 & 16.6 & 8.0 & 14.7 \\
\hline
\end{tabular}

Table 5. Concentration of the calcium ions in the coma of meteor 690803.

\begin{tabular}{|c|c|c|c|c|c|c|c|c|c|c|}
\hline Points & $I_{\mathrm{K}} / I_{\mathrm{H}}$ & $\begin{array}{l}I_{\mathrm{H}} \\
(13)\end{array}$ & $\log (N / \xi)_{\text {CaII }}$ & $D_{\mathrm{H}}$ & $\begin{array}{l}J \xi_{\text {CaII }} \\
(15)\end{array}$ & $\begin{array}{l}J \\
(10)\end{array}$ & $T$ & $\log N_{\text {CaII }}$ & $\begin{array}{l}b \\
\mathrm{~m}\end{array}$ & $\log n_{\text {CaII }}$ \\
\hline 2 & 1.26 & 0.5 & 12.2 & 31 & 0.1 & 0.1 & 1762 & 17.2 & 18.0 & 13.9 \\
\hline 3 & 1.78 & 4.0 & 11.2 & 124 & 5.0 & 3.5 & 2116 & 16.2 & 18.0 & 12.9 \\
\hline 4 & 1.32 & 63.0 & 12.0 & 35 & 22.0 & 16.0 & 2317 & 17.0 & 18.0 & 13.7 \\
\hline 5 & 1.12 & 0.2 & 13.0 & 21 & 0.04 & 0.03 & 1649 & 18.0 & 18.0 & 14.7 \\
\hline 6 & 1.26 & 1.0 & 12.2 & 31 & 0.3 & 0.2 & 1823 & 17.2 & 18.0 & 13.9 \\
\hline 7 & 1.26 & 11.0 & 12.2 & 31 & 3.4 & 2.4 & 2071 & 17.2 & 18.0 & 13.9 \\
\hline
\end{tabular}

Table 6. Concentration of the calcium ions in the coma of meteor 830633.

\begin{tabular}{|c|c|c|c|c|c|c|c|c|c|c|}
\hline Points & $I_{\mathrm{K}} / I_{\mathrm{H}}$ & $\begin{array}{l}I_{\mathrm{H}} \\
(10)\end{array}$ & $\log (N / \xi)_{\text {CaII }}$ & $D_{\mathrm{H}}$ & $\begin{array}{l}J \xi_{\text {CaII }} \\
(12)\end{array}$ & $\begin{array}{l}J \\
(6)\end{array}$ & $T$ & $\log N_{\text {CaII }}$ & $\begin{array}{l}b \\
\mathrm{~m}\end{array}$ & $\log n_{\text {CaII }}$ \\
\hline 2 & 1.28 & 2.2 & 12.1 & 33 & 0.7 & 5.3 & 1400 & 17.1 & 9.0 & 14.1 \\
\hline 4 & 1.17 & 4.9 & 12.5 & 26 & 1.3 & 9.0 & 1430 & 17.6 & 16.0 & 14.4 \\
\hline 5 & 1.23 & 3.7 & 12.3 & 29 & 1.1 & 7.7 & 1421 & 17.3 & 14.0 & 14.5 \\
\hline 6 & 1.28 & 2.2 & 12.1 & 34 & 0.8 & 5.4 & 1402 & 17.1 & 11.0 & 14.0 \\
\hline
\end{tabular}


P. B. Babadzhanov and G. I. Kokhirova: Calcium ions in a meteor coma, Online Material p 4

Table 7. Concentration of the calcium ions in the coma of meteor 830811 .

\begin{tabular}{|c|c|c|c|c|c|c|c|c|c|c|}
\hline Points & $I_{\mathrm{K}} / I_{\mathrm{H}}$ & $\begin{array}{l}I_{\mathrm{H}} \\
(12)\end{array}$ & $\log (N / \xi)_{\text {CaII }}$ & $D_{\mathrm{H}}$ & $\begin{array}{l}J \xi_{\text {CaII }} \\
(13)\end{array}$ & $\begin{array}{l}J \\
(8)\end{array}$ & $T$ & $\log N_{\text {CaII }}$ & $\begin{array}{l}b \\
\mathrm{~m}\end{array}$ & $\log n_{\text {CaII }}$ \\
\hline 1 & 1.49 & 0.5 & 11.7 & 48 & 2.2 & 1.6 & 1610 & 16.8 & 7.0 & 13.9 \\
\hline 2 & 1.16 & 1.0 & 12.6 & 25 & 2.4 & 1.7 & 1617 & 17.6 & 7.0 & 14.7 \\
\hline 3 & 1.08 & 1.5 & 13.3 & 18 & 2.7 & 1.9 & 1625 & 18.3 & 7.5 & 15.4 \\
\hline 4 & 1.42 & 1.6 & 11.8 & 41 & 6.5 & 4.6 & 1691 & 16.9 & 8.0 & 14.0 \\
\hline 5 & 1.42 & 1.7 & 11.8 & 41 & 6.8 & 4.9 & 1695 & 16.9 & 8.0 & 14.0 \\
\hline 6 & 1.15 & 2.0 & 12.6 & 25 & 5.0 & 3.5 & 1670 & 17.7 & 8.0 & 14.8 \\
\hline 8 & 1.28 & 1.8 & 12.1 & 32 & 5.8 & 4.2 & 1682 & 17.2 & 8.5 & 14.3 \\
\hline 9 & 1.24 & 1.7 & 12.2 & 30 & 5.2 & 3.7 & 1674 & 17.3 & 9.0 & 14.3 \\
\hline 10 & 1.24 & 1.7 & 12.2 & 30 & 5.0 & 3.6 & 1670 & 17.3 & 9.0 & 14.3 \\
\hline 11 & 1.36 & 1.6 & 11.9 & 38 & 6.0 & 4.3 & 1685 & 16.9 & 9.0 & 13.9 \\
\hline 12 & 1.24 & 1.5 & 12.2 & 30 & 4.5 & 3.2 & 1663 & 17.3 & 8.5 & 14.4 \\
\hline 13 & 1.24 & 1.4 & 12.2 & 30 & 4.1 & 3.0 & 1656 & 17.3 & 8.0 & 14.4 \\
\hline 14 & 1.35 & 1.1 & 11.9 & 38 & 4.4 & 3.1 & 1660 & 16.9 & 8.0 & 14.0 \\
\hline 15 & 1.08 & 0.8 & 13.3 & 18 & 1.5 & 1.1 & 1583 & 18.3 & 7.0 & 15.4 \\
\hline 18 & 1.08 & 0.2 & 13.3 & 18 & 0.2 & 0.2 & 1467 & 18.3 & 7.0 & 15.4 \\
\hline
\end{tabular}

Table 8. Concentration of the calcium ions in the coma of meteor 830953.

\begin{tabular}{cccccccccccc}
\hline \hline Points & $I_{\mathrm{K}} / I_{\mathrm{H}}$ & $I_{\mathrm{H}}$ & $\log (N / \xi)_{\text {CaII }} D_{\mathrm{H}}$ & \multicolumn{2}{c}{$J \xi_{\text {CaII }} J$} & $T$ & $\log N_{\text {CaII }} b$ & $\log n_{\text {CaII }}$ \\
& & $(9)$ & & & $(11)$ & $(6)$ & & & $\mathrm{m}$ & \\
\hline 1 & 1.09 & 26.0 & 13.3 & 18 & 4.7 & 3.4 & 1376 & 18.3 & 8.7 & 15.4 \\
2 & 1.25 & 15.0 & 12.2 & 30 & 4.5 & 3.2 & 1374 & 17.2 & 7.5 & 14.3 \\
3 & 1.29 & 13.0 & 12.1 & 32 & 4.1 & 2.9 & 1369 & 17.1 & 7.0 & 14.2 \\
4 & 1.37 & 11.0 & 11.9 & 38 & 4.3 & 3.1 & 1372 & 16.9 & 8.5 & 14.1 \\
5 & 1.46 & 9.5 & 11.7 & 48 & 4.5 & 3.2 & 1374 & 16.8 & 9.5 & 13.8 \\
6 & 1.57 & 6.4 & 11.6 & 56 & 3.6 & 2.5 & 1362 & 16.7 & 8.5 & 13.7 \\
7 & 1.63 & 5.3 & 11.5 & 80 & 4.3 & 3.1 & 1371 & 16.5 & 9.0 & 13.5 \\
8 & 1.65 & 3.8 & 11.4 & 90 & 3.5 & 2.5 & 1360 & 16.5 & 9.5 & 13.4 \\
9 & 1.89 & 1.8 & 10.8 & 190 & 3.4 & 2.4 & 1360 & 15.8 & 11.0 & 12.8 \\
10 & 1.57 & 1.4 & 11.6 & 56 & 0.8 & 0.5 & 1288 & 16.7 & 12.0 & 13.7 \\
12 & 1.89 & 0.8 & 10.8 & 190 & 1.5 & 1.1 & 1319 & 16.5 & 10.0 & 13.4 \\
\hline
\end{tabular}


P. B. Babadzhanov and G. I. Kokhirova: Calcium ions in a meteor coma, Online Material p 5

Table 9. Concentration of the calcium ions in the coma of meteor 900315.

\begin{tabular}{|c|c|c|c|c|c|c|c|c|c|c|}
\hline Points & $I_{\mathrm{K}} / I_{\mathrm{H}}$ & $\begin{array}{l}I_{\mathrm{H}} \\
(11)\end{array}$ & $\log (N / \xi)_{\mathrm{CaII}}$ & $D_{\mathrm{H}}$ & $\begin{array}{l}J \xi_{\text {CaII }} \\
(13)\end{array}$ & $\begin{array}{l}J \\
(7)\end{array}$ & $T$ & $\log N_{\mathrm{CaII}}$ & $\begin{array}{l}b \\
\mathrm{~m}\end{array}$ & $\log n_{\text {CaII }}$ \\
\hline & & & & First & flare & & & & & \\
\hline 1 & 1.27 & 0.6 & 12.5 & 32 & 0.2 & 1.9 & 1472 & 17.2 & 9.0 & 14.2 \\
\hline 2 & 1.46 & 2.1 & 11.7 & 48 & 1.0 & 9.3 & 1574 & 16.8 & 9.0 & 13.8 \\
\hline 3 & 1.16 & 4.9 & 12.7 & 24 & 1.2 & 0.1 & 1583 & 17.8 & 9.0 & 14.8 \\
\hline 4 & 1.10 & 7.1 & 13.3 & 18 & 1.3 & 0.1 & 1588 & 18.3 & 9.0 & 15.3 \\
\hline 5 & 1.10 & 5.6 & 13.3 & 18 & 1.0 & 9.2 & 1573 & 18.3 & 9.0 & 15.3 \\
\hline 6 & 1.16 & 1.5 & 12.7 & 24 & 0.4 & 3.3 & 1507 & 17.8 & 9.0 & 14.8 \\
\hline \multirow[t]{2}{*}{7} & 1.83 & 0.5 & 11.1 & 140 & 0.7 & 6.5 & 1550 & 16.1 & 9.0 & 13.1 \\
\hline & & & & Second & flare & & & & & \\
\hline 2 & 1.97 & 3.9 & 10.2 & - & - & - & - & 15.2 & 9.0 & 12.2 \\
\hline 3 & 1.20 & 1.4 & 12.4 & 27 & 0.3 & 2.7 & 1495 & 17.4 & 9.0 & 14.4 \\
\hline
\end{tabular}

